\title{
Hypergeometric function expressions for the molecule-micropore Lennard--Jones potential
}

\author{
Guangfeng Jiang* \\ Jianghua Zhang* \\ XianRen Zhang ${ }^{\dagger}$ \\ Wenchuan Wang ${ }^{\dagger}$
}

(Received 10 October 2003; revised 3 August 2004)

\begin{abstract}
We present hypergeometric function expressions for the moleculemicropore Lennard-Jones potential in cylindrical pores, in which the cylindrical wall can be a surface, or have thickness or have infinite thickness. These expressions are useful in theoretical study and computer simulations of fluids in micropore of circular cross sections.
\end{abstract}

${ }^{*}$ Department of mathematics and information science, Mailbox 66, Faculty of Science, Beijing university of chemical technology, Beisanhuandonglu 15, Beijing 100029, P. R. China. mailto: jianggf@mail.buct.edu.cn, mailto:menc222@sina.com $\dagger$ College of Chemical Engineering, Beijing university of chemical technology, Beisanhuandonglu 15, Beijing 100029, P. R. China. mailto:wangwc@mail .buct.edu.cn, mailto:zhangxr@mail. buct.edu.cn

See http://anziamj.austms.org.au/V46/E/Jiang/home.html for this article, (C) Austral. Mathematical Soc. 2004. Published October 5, 2004; amended December 1, 2004. ISSN 1446-8735 


\section{Contents}

1 Introduction

E71

2 The cylindrical surface wall without thickness

E73

3 The cylindrical pore with infinite thickness

E77

References

E84

\section{Introduction}

Microporous and mesoporous materials, such as zeolites, aluminosilicates, activated carbon, and pillared clays, have a wide range of industrial applications, including heterogeneous catalysis, purification of gases and water streams, and storage of gaseous fuels. Recently there has been great interest in theoretical and computer simulation studies of the equilibrium and transport properties of fluids confined inside micropores. The interaction between the fluid molecules and the micropore walls have been taken into account by introducing various interatomic potentials from the view point of quantum and statistical mechanics. The main idea is as follows. In colloidal system, the interatomic potential consists of an attractive contribution from van der Walls forces and repulsive part due to electrostatic effects between the charged particles.

The most widely used interatomic potential is the so called Lennard-Jones $p-q$ potential $[1]$.

$$
E_{p, q}=\frac{q \epsilon}{q-p}\left(\frac{q}{p}\right)^{p /(q-p)}\left[\left(\frac{\sigma}{r}\right)^{q}-\left(\frac{\sigma}{r}\right)^{p}\right]
$$

where $r$ is the interatomic separation, $\sigma$ the separation at which the potential becomes 0 , and $\epsilon$ the depth of the primary well. The two adjustable 
parameters $\sigma$ and $\epsilon$ are actually determined on the basis of experiments.

The Lennard-Jones potential contains an attractive part due to van der Waals forces and a repulsive contribution known as Born forces.

The parameter pair $(p, q)$ is often chosen to be $(6, q)(8 \leq q \leq 20)$ and $(4,20)$. For this reason, we assume in this article that $p$ and $q$ are even numbers with $4 \leq p<q$.

Denote by

$$
C_{p, q}=\frac{q \epsilon}{q-p}\left(\frac{q}{p}\right)^{p /(q-p)} .
$$

For example, $C_{6,12}=4 \epsilon$.

If the atoms up to and on the wall of the cylindrical pore are distributed continuously, the interaction energy $U=U_{p, q}$ of the test fluid atom with the wall of volume $\Omega$ and number density $N$ of the wall molecules is given by the interaction over $\Omega$, we have

$$
U=U_{p, q}=N \int_{\Omega} E_{p, q} d \Omega .
$$

To evaluate this integral, one meets a kind of elliptic type integrals. In this article, we express $U=U_{p, q}$ by hypergeometric functions for $\Omega$ to be the following objects:

1. a cylindrical surface with infinite length and without thickness (see formula (8));

2. the complement of a cylindrical pore in the three dimensional Euclidean space $\mathbb{R}^{3}$ with infinite length (see the formula in Remark 7);

3. the wall of a cylindrical pore with infinite length and finite thickness (see the formula in Remark 8).

Note that the last case can be deduced from the second case. 


\section{The cylindrical surface wall without thickness}

In this section we assume the wall of the cylindrical pore is a cylinder surface $S$ with infinite length and without thickness. The interaction energy $U_{p, q}$ of the test fluid atom with the surface of area $S$ and number density $N$ of the wall molecules is given by the following integral over $S$ :

$$
U_{p, q}=N C_{p, q} \iint_{S}\left[\left(\frac{\sigma}{r}\right)^{q}-\left(\frac{\sigma}{r}\right)^{p}\right] d S .
$$

We explain the notations in the this integral (see Figure 1). The test fluid atom sits at the origin $O_{T}$ of the coordinate in $\mathbb{R}^{3}$, the $z$ axis is parallel to the axis of the cylinder, choose the $x$ axis such that the symmetric center $O$ of the cylinder is on the negative part of the $x$ axis. Let $\left|O O_{T}\right|=\rho, R$ is the radius of the cylinder. For $P(x, y, z)$ on the cylinder $S$, let $A(x, y, 0)$, $\varphi=\angle\left(\overrightarrow{O_{T} A}, x-\right.$ axis $)$, and $\psi=\angle\left(\overrightarrow{O O_{T}}, \overrightarrow{O A}\right)$. Denote by

$$
r^{2}=\left|O_{T} P\right|^{2}=z^{2}+\tilde{r}^{2},
$$

where

$$
\tilde{r}^{2}=\left|O_{T} A\right|=\rho^{2}+R^{2}-2 \rho R \cos \psi .
$$

By symmetry properties, we have

$$
U_{p, q}=4 N R C_{p, q} \int_{0}^{\pi} d \psi \int_{0}^{+\infty}\left[\left(\frac{\sigma^{2}}{\tilde{r}^{2}+z^{2}}\right)^{q / 2}-\left(\frac{\sigma^{2}}{\tilde{r}^{2}+z^{2}}\right)^{p / 2}\right] d z
$$

Recall that $p$ and $q$ are even numbers. Since

$$
\int_{0}^{+\infty} \frac{1}{\left(a^{2}+z^{2}\right)^{s}} d z=\frac{\pi}{2} \cdot \frac{(2 s-3) ! !}{(2 s-2) ! !} \cdot \frac{1}{a^{2 s-1}},
$$




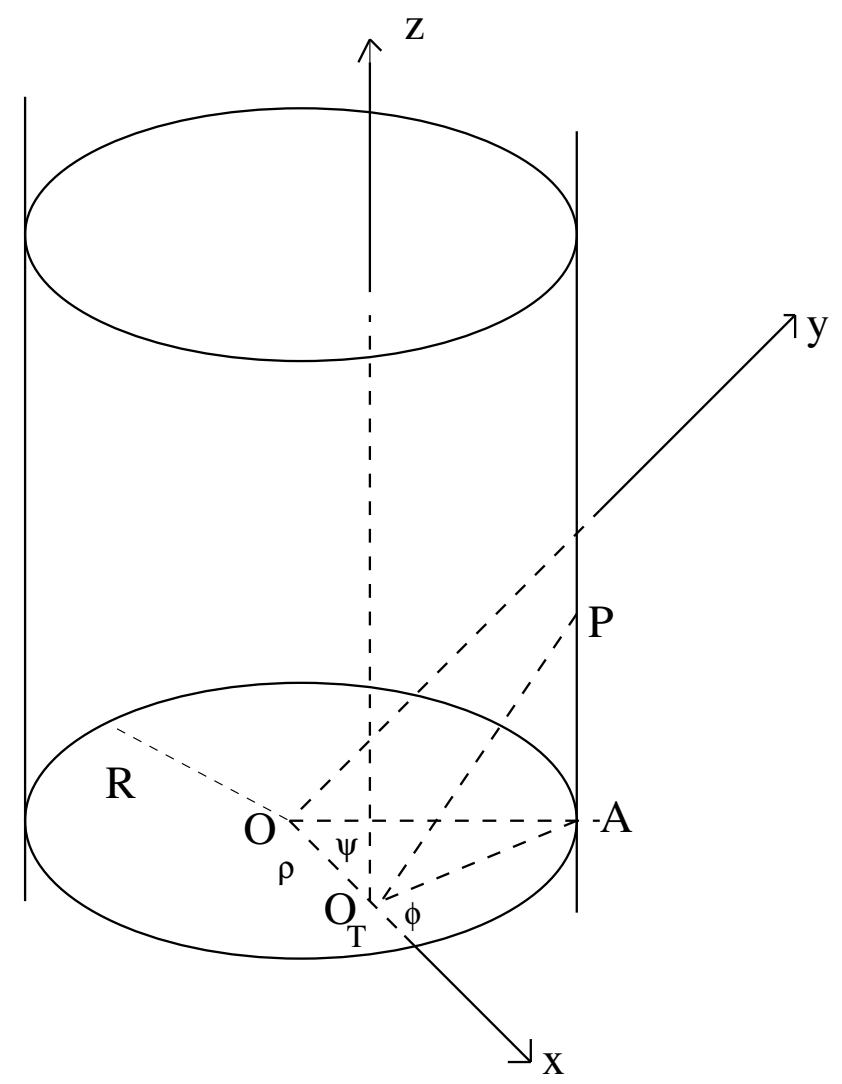

Figure 1: 
we have

$$
U_{p, q}=4 N R C_{p, q}\left[\frac{\pi}{2} \cdot \frac{(q-3) ! !}{(q-2) ! !} \sigma^{q} \int_{0}^{\pi} \frac{d \psi}{\tilde{r}^{q-1}}-\frac{\pi}{2} \cdot \frac{(p-3) ! !}{(p-2) ! !} \sigma^{p} \int_{0}^{\pi} \frac{d \psi}{\tilde{r}^{p-1}}\right] .
$$

We have to evaluate the following integral

$$
\begin{aligned}
\tilde{J}_{2 s} & =\int_{0}^{\pi} \frac{d \psi}{\tilde{r}^{2 s-1}}=\int_{0}^{\pi} \frac{d \psi}{\left(\rho^{2}+R^{2}-2 \rho R \cos \psi\right)^{(2 s-1) / 2}} \\
& =\frac{2}{(\rho+R)^{2 s-1}} \int_{0}^{\pi / 2} \frac{d \theta}{\left(1-k^{2} \sin ^{2} \theta\right)^{(2 s-1) / 2}}
\end{aligned}
$$

where $k=2 \sqrt{\rho R} /(\rho+R)$.

Let $t=\sin ^{2} \theta$, then $d t=2 \sin \theta \cos \theta d \theta=2 \sqrt{t} \sqrt{1-t} d \theta$. From (5) we have

$$
\tilde{J}_{2 s}=\frac{1}{(\rho+R)^{2 s-1}} \int_{0}^{1} t^{-1 / 2}(1-t)^{-1 / 2}\left(1-k^{2} t\right)^{-(2 s-1) / 2} d t .
$$

By integral representation of hypergeometric function [2], we have

$$
\tilde{J}_{2 s}=\frac{\pi}{(\rho+R)^{2 s-1}} F\left(\frac{2 s-1}{2}, \frac{1}{2} ; 1 ; k^{2}\right) .
$$

Hence,

$$
\begin{gathered}
U_{p, q}=2 \pi^{2} N R C_{p, q}\left[\frac{(q-3) ! !}{(q-2) ! !} \frac{\sigma^{q}}{(\rho+R)^{q-1}} F\left(\frac{q-1}{2}, \frac{1}{2} ; 1 ; k^{2}\right)\right. \\
\left.-\frac{(p-3) ! !}{(p-2) ! !} \frac{\sigma^{p}}{(\rho+R)^{p-1}} F\left(\frac{p-1}{2}, \frac{1}{2} ; 1 ; k^{2}\right)\right]
\end{gathered}
$$

where $k^{2}=4 \rho R /(\rho+R)^{2}$. 


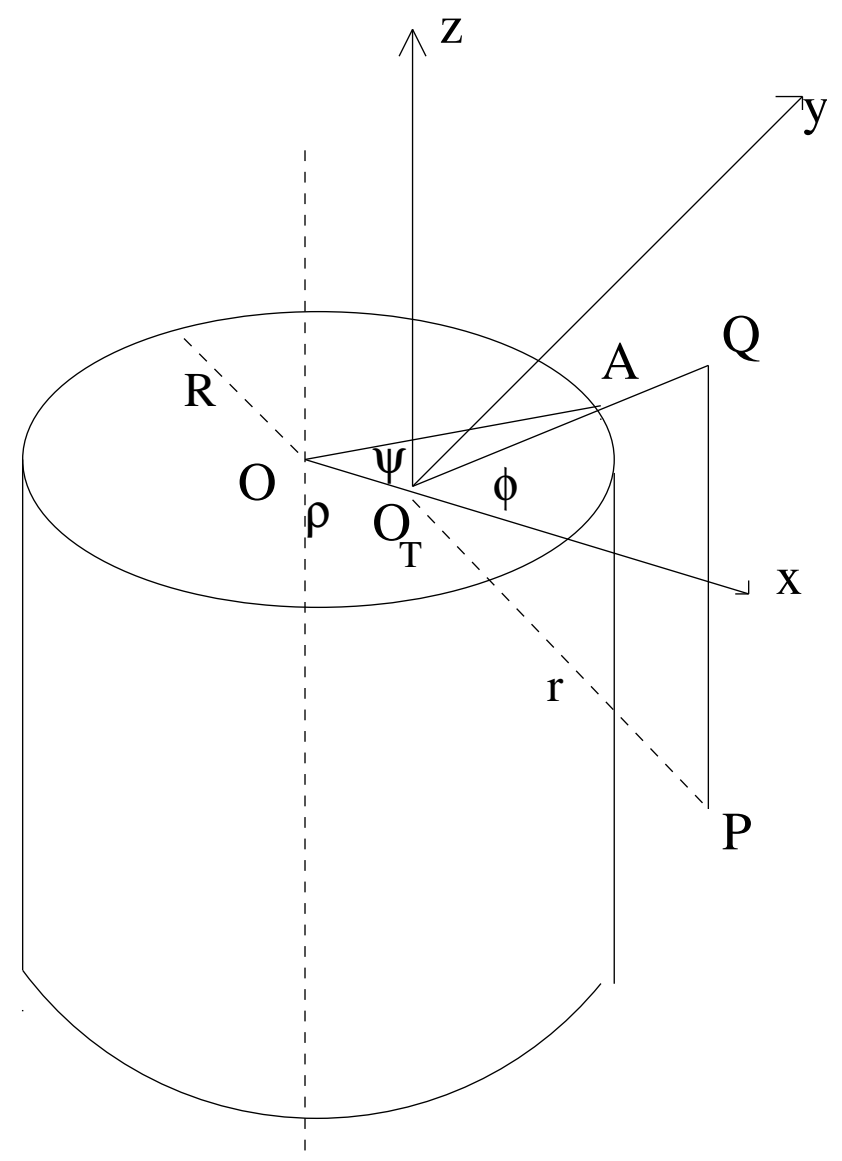

Figure 2: 


\section{The cylindrical pore with infinite thickness}

In this section, we consider a test atom in a cylindrical pore, and the thickness of the wall is infinite. Suppose that the atoms of the wall are distributed continuously and uniformly within the whole region of the wall. Let the test atom sit at the origin $O_{T}$, the $z$ axis be parallel to the axis of the cylindrical surface, and, the intersection $O$ of the $x$ axis and the axis of the cylindrical surface be on the negative part of the $x$ axis. See Figure 2.

Denote by $\Omega$ the complement of the cylindrical pore in the three dimensional Euclidean space $\mathbb{R}^{3}$. Then the Lennard-Jones potential is

$$
U_{p, q}=N \iint_{\Omega} E_{p, q} d \Omega=2 N C_{p, q} \iint_{D} d x d y \int_{0}^{+\infty}\left[\left(\frac{\sigma}{r}\right)^{q}-\left(\frac{\sigma}{r}\right)^{p}\right] d z,
$$

where $D:=\Omega \cap\{z=0\}, r=\sqrt{x^{2}+y^{2}+z^{2}}, \tilde{r}=\left|O_{T} Q\right|=\sqrt{x^{2}+y^{2}}$. By (3), we have

$$
U_{p, q}=N \pi C_{p, q}\left[\sigma^{q} \frac{(q-3) ! !}{(q-2) ! !} \iint_{D} \frac{d x d y}{\tilde{r}^{q-1}}-\sigma^{p} \frac{(p-3) ! !}{(p-2) ! !} \iint_{D} \frac{d x d y}{\tilde{r}^{p-1}}\right] .
$$

Since we assume $p$ and $q$ are even number, it is enough to prove the following lemma.

Lemma 1 By the above notation,

$$
T_{2 s-1}:=\iint_{D} \frac{d x d y}{\tilde{r}^{2 s-1}}=\frac{2}{2 s-3} \int_{0}^{\pi} \frac{1}{r_{\phi}^{2 s-3}} d \phi,
$$

where $r_{\phi}=\left|O_{T} A\right|$. 


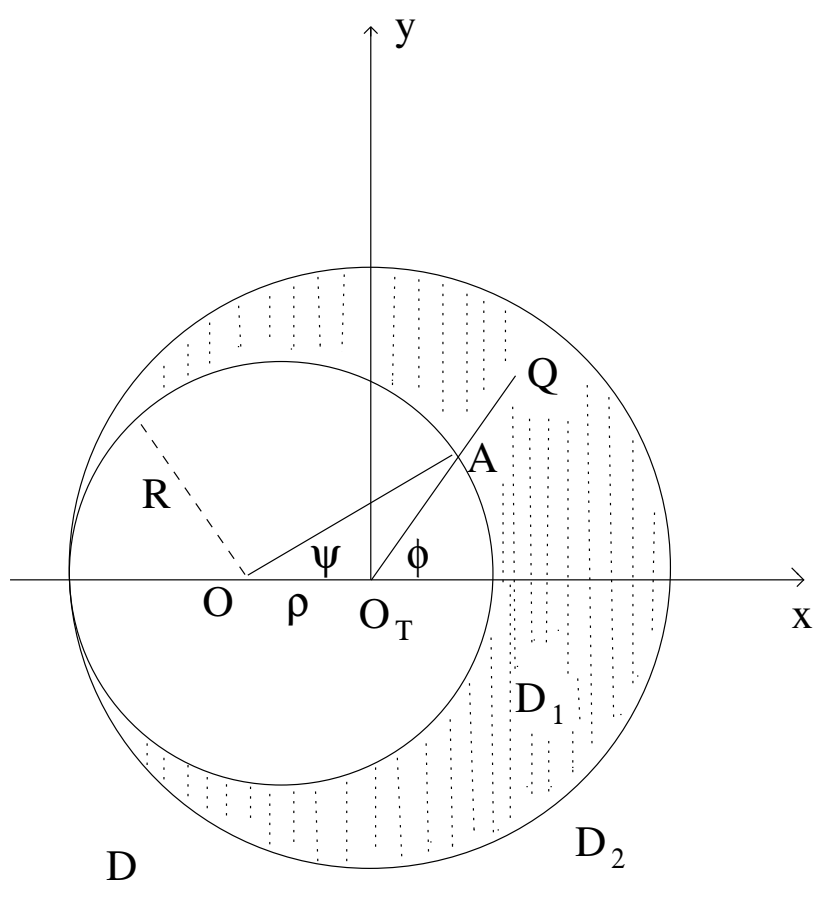

FIGURE 3: 
Proof: We divide $D$ into two parts (see Figure 3):

$$
D_{1}:=\left\{(x, y) \mid x^{2}+y^{2} \leq(R+\rho)^{2}\right\} \cap D, \quad D_{2}:=D \backslash D_{1},
$$

where $\rho=\left|O O_{t}\right|$. By using polar coordinates, one easily evaluates the integral on $D_{2}$ :

$$
\iint_{D_{2}} \frac{d x d y}{\left(x^{2}+y^{2}\right)^{(2 s-1) / 2}}=\int_{0}^{2 \pi} d \theta \int_{\rho+R}^{+\infty} \frac{\tilde{r} d \tilde{r}}{\tilde{r}^{2 s-1}}=\frac{2 \pi}{2 s-3} \frac{1}{(\rho+R)^{2 s-3}} .
$$

Similarly

$$
\begin{aligned}
\iint_{D_{1}} \frac{d x d y}{\left(x^{2}+y^{2}\right)^{(2 s-1) / 2}} & =2 \int_{0}^{\pi} d \phi \int_{r_{\phi}}^{\rho+R} \frac{\tilde{r} d \tilde{r}}{\tilde{r}^{2 s-1}} \\
& =\frac{2}{2 s-3} \int_{0}^{\pi}\left[\frac{1}{r_{\phi}^{2 s-3}}-\frac{1}{(\rho+R)^{2 s-3}}\right] d \phi .
\end{aligned}
$$

Lemma 2 Let $k:=\rho / R$, then

$$
T_{2 s-1}=\frac{2}{2 s-3} \frac{R^{2 s-3}}{\left(R^{2}-\rho^{2}\right)^{2 s-3}} \int_{0}^{\pi}\left(\sqrt{1-k^{2} \sin ^{2} \phi}+k \cos \phi\right)^{2 s-3} d \phi .
$$

Proof: Since $r_{\phi}>0$, and

$$
r_{\phi}^{2}+2 \rho r_{\phi} \cos \phi+\rho^{2}-R^{2}=0,
$$

we have

$$
r_{\phi}=-\rho \cos \phi+\sqrt{R^{2}-\rho^{2} \sin ^{2} \phi}=R\left(\sqrt{1-k^{2} \sin ^{2} \phi}-k \cos \phi\right) .
$$

Substituting this into formula (11) in Lemma 1, we obtain formula (12). 
Theorem 3 For integer $n \geq 1$, denote by

$$
I_{2 n-1}=\int_{0}^{\pi}\left(\sqrt{1-k^{2} \sin ^{2} \phi}+k \cos \phi\right)^{2 n-1} d \phi
$$

then

$$
I_{2 n-1}=\pi F\left(-\frac{2 n-1}{2},-\frac{2 n-3}{2} ; 1 ; k^{2}\right)
$$

We divide the proof of Theorem 3 into the following three lemmas. In the following, we agree that $(-1) ! !=1,0 !=1$ and $\left(\begin{array}{l}n \\ 0\end{array}\right)=1$.

Lemma 4

$$
I_{2 n-1}=2 \sum_{\mu=0}^{n-1}\left(\begin{array}{c}
2 n-1 \\
2 \mu
\end{array}\right) k^{2 \mu} \int_{0}^{\frac{\pi}{2}}\left(1-k^{2} \sin ^{2} \phi\right)^{\frac{2 n-1-2 \mu}{2}}(\cos \phi)^{2 \mu} d \phi .
$$

Proof: Note

$$
\begin{aligned}
I_{2 n-1}= & \int_{0}^{\frac{\pi}{2}}\left(\sqrt{1-k^{2} \sin ^{2} \phi}+k \cos \phi\right)^{2 n-1} d \phi \\
& +\int_{\frac{\pi}{2}}^{\pi}\left(\sqrt{1-k^{2} \sin ^{2} \phi}+k \cos \phi\right)^{2 n-1} d \phi .
\end{aligned}
$$

For the second integral, let $t=\pi-\phi$, we have

$$
\begin{aligned}
I_{2 n-1}=\int_{0}^{\frac{\pi}{2}} & {\left[\left(\sqrt{1-k^{2} \sin ^{2} \phi}+k \cos \phi\right)^{2 n-1}\right.} \\
+ & \left.\left(\sqrt{1-k^{2} \sin ^{2} \phi}-k \cos \phi\right)^{2 n-1}\right] d \phi
\end{aligned}
$$




$$
\begin{aligned}
&=2 \int_{0}^{\frac{\pi}{2}} {\left[\left(\sqrt{1-k^{2} \sin ^{2} \phi}+k \cos \phi\right)^{2 n-1}\right.} \\
&\left.+\left(\sqrt{1-k^{2} \sin ^{2} \phi}-k \cos \phi\right)^{2 n-1}\right] d \phi \\
&=2 \int_{0}^{\frac{\pi}{2}}\left[\sum_{\mu=0}^{n-1}\left(\begin{array}{c}
2 n-1 \\
2 \mu
\end{array}\right)\left(\sqrt{1-k^{2} \sin ^{2} \phi}\right)^{2 n-1-2 \mu}(k \cos \phi)^{2 \mu}\right] d \phi \\
&=2 \sum_{\mu=0}^{n-1}\left(\begin{array}{c}
2 n-1 \\
2 \mu
\end{array}\right) k^{2 \mu} \int_{0}^{\frac{\pi}{2}}\left(1-k^{2} \sin ^{2} \phi\right)^{(2 n-1-2 \mu) / 2}(\cos \phi)^{2 \mu} d \phi .
\end{aligned}
$$

\section{Lemma 5}

$$
\begin{aligned}
I_{2 n-1}= & \pi F\left(-n+\frac{1}{2}, \frac{1}{2} ; 1 ; k^{2}\right) \\
+ & \pi \sum_{\mu=1}^{n-1}\left(\begin{array}{c}
n-1 \\
\mu
\end{array}\right) \frac{(2 n-1)(2 n-3) \cdots(2 n-2 \mu+1)}{2^{\mu} \mu !} \\
& \times k^{2 \mu} F\left(-n+\mu+\frac{1}{2}, \frac{1}{2} ; \mu+1 ; k^{2}\right) .
\end{aligned}
$$

Proof: Substitute $t=\sin ^{2} \phi$ into formula (15), we have

$$
I_{2 n-1}=\sum_{\mu=0}^{n-1}\left(\begin{array}{c}
2 n-1 \\
2 \mu
\end{array}\right) k^{2 \mu} \int_{0}^{1} t^{-\frac{1}{2}}(1-t)^{\mu-\frac{1}{2}}\left(1-k^{2} t\right)^{n-\mu-\frac{1}{2}} d t .
$$

By the integral representation of hypergeometric function [2]

$$
F(\alpha, \beta ; \gamma ; z)=\frac{1}{B(\beta, \gamma-\beta)} \int_{0}^{1} t^{\beta-1}(1-t)^{\gamma-\beta-1}(1-t z)^{-\alpha} d t
$$


where $\Re \gamma>\Re \beta>0$, and $B(-,-)$ is the beta function.

Let $\alpha=-n+\mu+\frac{1}{2}, \beta=\frac{1}{2}, \gamma=\mu+1$, and $z=k^{2}$, we have

$$
\begin{aligned}
& \int_{0}^{1} t^{-\frac{1}{2}}(1-t)^{\mu-1 / 2}\left(1-t k^{2}\right)^{n-\mu-1 / 2} d t \\
& =B\left(\frac{1}{2}, \mu+\frac{1}{2}\right) F\left(-n+\mu+\frac{1}{2}, \frac{1}{2} ; \mu+1 ; k^{2}\right) .
\end{aligned}
$$

Then

$$
I_{2 n-1}=\sum_{\mu=0}^{n-1}\left(\begin{array}{c}
2 n-1 \\
2 \mu
\end{array}\right) k^{2 \mu} B\left(\frac{1}{2}, \mu+\frac{1}{2}\right) F\left(-n+\mu+\frac{1}{2}, \frac{1}{2} ; \mu+1 ; k^{2}\right) .
$$

Since

$$
B\left(\frac{1}{2}, \mu+\frac{1}{2}\right)=\frac{\Gamma\left(\frac{1}{2}\right) \Gamma\left(\mu+\frac{1}{2}\right)}{\Gamma(\mu+1)}=\frac{\pi(2 \mu-1) ! !}{2^{\mu} \mu !}
$$

For $\mu \geq 1$

$$
\left(\begin{array}{c}
2 n-1 \\
2 \mu
\end{array}\right)=\left(\begin{array}{c}
n-1 \\
\mu
\end{array}\right) \frac{(2 n-1)(2 n-3) \cdots(2 n-2 \mu+1)}{(2 \mu-1) ! !} .
$$

And

$$
\left(\begin{array}{c}
2 n-1 \\
2 \mu
\end{array}\right) B\left(\frac{1}{2}, \mu+\frac{1}{2}\right)=\pi\left(\begin{array}{c}
n-1 \\
\mu
\end{array}\right) \frac{(2 n-1)(2 n-3) \cdots(2 n-2 \mu+1)}{2^{\mu} \mu !} .
$$

This proves the lemma.

\section{Lemma 6}

$$
\begin{aligned}
F & \left(-n+\frac{1}{2},-n+1+\frac{1}{2} ; 1 ; k^{2}\right) \\
= & \sum_{\mu=0}^{n-1}\left(\begin{array}{c}
n-1 \\
\mu
\end{array}\right) \frac{(2 n-1)(2 n-3) \cdots(2 n-2 \mu+1)}{2^{\mu} \mu !} \\
& \times k^{2 \mu} F\left(-n+\mu+\frac{1}{2}, \frac{1}{2} ; \mu+1 ; k^{2}\right)
\end{aligned}
$$


Proof: We expand the left side of (16) by using Gauss recursion formula [2]

$$
\gamma F(\alpha, \beta ; \gamma ; z)-\gamma F(\alpha, \beta+1 ; \gamma ; z)+\alpha z F(\alpha+1, \beta+1 ; \gamma+1 ; z),
$$

that is,

$$
F(\alpha, \beta ; \gamma ; z)=F(\alpha, \beta+1 ; \gamma ; z)-\frac{\alpha z}{\gamma} F(\alpha+1, \beta+1 ; \gamma+1 ; z) .
$$

We stop the expansion process when the second parameter $\beta=\frac{1}{2}$ in every hypergeometric function. Then we consider the coefficients of the terms $k^{2 \mu} F\left(-n+\mu+\frac{1}{2}, \frac{1}{2} ; \mu+1 ; k^{2}\right)$ for each $\mu=0,1,2, \ldots, n-1$ in the expansion before collecting them together. We find that for fixed $n$, the coefficients of the term $k^{2 \mu} F\left(-n+\mu+\frac{1}{2}, \frac{1}{2} ; \mu+1 ; k^{2}\right)$ are equal to

$$
\frac{(2 n-1)(2 n-3) \cdots(2 n-2 \mu+1)}{2^{\mu} \mu !},
$$

for each $\mu=0,1,2, \ldots, n-1$.

Moreover, in the expansion, the term

$$
\frac{(2 n-1)(2 n-3) \cdots(2 n-2 \mu+1)}{2^{\mu} \mu !} k^{2 \mu} F\left(-n+\mu+\frac{1}{2}, \frac{1}{2} ; \mu+1 ; k^{2}\right)
$$

appears as many as $\left(\begin{array}{c}n-1 \\ \mu\end{array}\right)$ times, $\mu=0,1,2, \ldots, n-1$. This finishes the proof of the lemma.

Remark 7 By Lemmas 1 and 2 and Theorem 3 we have

$$
\begin{aligned}
U_{p, q}=2 N & \pi^{2} C_{p, q}\left[\frac{(q-5) ! !}{(q-2) ! !} \frac{\sigma^{q} R^{q-3}}{\left(R^{2}-\rho^{2}\right)^{q-3}} F\left(\frac{3-q}{2}, \frac{5-q}{2} ; 1 ; k^{2}\right)\right. \\
& \left.-\frac{(p-5) ! !}{(p-2) ! !} \frac{\sigma^{p} R^{p-3}}{\left(R^{2}-\rho^{2}\right)^{p-3}} F\left(\frac{3-p}{2}, \frac{5-p}{2} ; 1 ; k^{2}\right)\right] .
\end{aligned}
$$


Remark 8 If wall of the cylindrical pore is of finite thickness, for example, the radii of inner and outer cylinders are $R_{1}<R_{2}$ respectively, the potential is

$$
U_{p, q}\left(R_{1}\right)-U_{p, q}\left(R_{2}\right)
$$

This was used in $[3,4]$.

Acknowledgment: This paper was supported by NSF of China under grant No.20236010

\section{References}

[1] D. L. Feke, N. D. Prabhu, J. Adin. Mann, Jr., J. Adin. Mann, A formulation of the short-Range repulsion between spherical colloidal particles, J. Phys. Chem. 88(1988) 5735-5739. E71

[2] Ryshik,I.M., Gradstein, I.S., Tables of series, Products, and integrals, Veb Deutscher Verlag der Wissenschaften: Berlin 1962. E75, E81, E83

[3] Zhang X., Wang W., A Potential Model for Cylindrical Pores, Chinese J. of Chem. Eng. 9(4) (2001) 348-353. E84

[4] Zhang X., Wang W., Jiang, G, A potential model for cylindrical pores with Lennard-Jones wall-fluid interaction, to appear. E84 